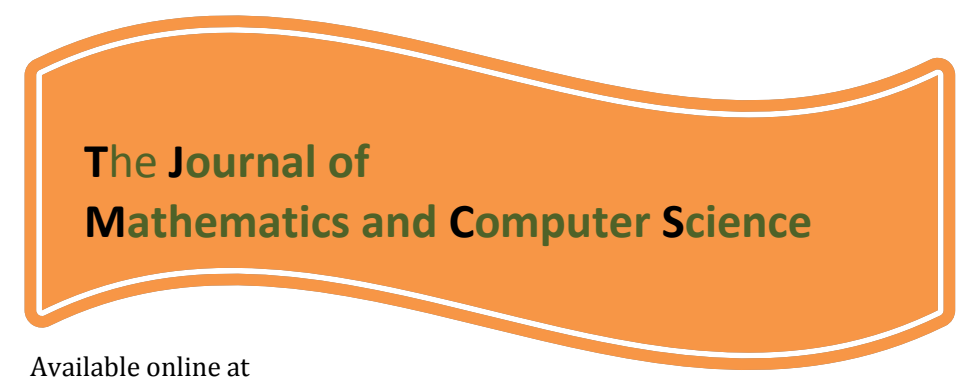

http://www.TJMCS.com

The Journal of Mathematics and Computer Science Vol .3 No.4 (2011) 396 - 402

\title{
Determining the objective value range for a class of interval convex optimization problems
}

\author{
Akbar Hashemi Borzabadi ${ }^{1}$, Leila Heidarian $^{2}$ \\ 1,2 School of Mathematics and Computer Science, Damghan University, Damghan, Iran. \\ borzabadi@du.ac.ir
}

Received: September 2011, Revised: November 2011

Online Publication: December 2011

\begin{abstract}
This paper generalizes a method of determining the objective value range of quadratic programming problems to a general class of interval convex programming ones, where all coefficients in objective function and constraints are interval numbers. The upper bound and lower bound of the objective values of the interval quadratic program is calculated by formulating a pair of two-level mathematical programs. Based on the duality theorem and by applying the variable transformation technique, the pair of two-level mathematical programs is transformed into conventional one-level convex programming problem. Solving the pair of convex programs produces the interval of the objective values of the problem. Numerical results confirms the procedure of the presented approach.
\end{abstract}

Keywords: Interval convex programming; Interval parameters; Two-level program.

\section{Introduction}

Recently, interval optimization problems as an extension of optimization problems with interval parameters and coefficients have been interested by many scientist. Various applications of these problems in study of uncertain systems and multiobjective programming problems and also their flexibility in providing different concept of solutions are some reasons to be concerned (see $[1,3,6,5]$ ). One can see in [9] and [10], attempts to 
examine the optimality conditions in some classes of these problems, considering a solution concept. One category of nonlinear optimization problems where the objective to be minimized and the constraints are both convex is called convex optimization problem. A class of interval convex optimization problems that the objective and constraints functions are the linear positive combination of convex functions and the combination coefficients are interval can be formulated as

$$
\begin{gathered}
\min _{\mathbf{x}} Z=\sum_{k=1}^{t} \bar{\gamma}_{k} f_{k}(\mathbf{x}), \\
\sum_{j=1}^{s} \bar{q}_{i j} g_{i j}(\mathbf{x}) \leq \overline{b_{i}}, i=1, \cdots, r, \\
\mathbf{x} \geq 0 .
\end{gathered}
$$

where $f_{k}(\mathbf{x}), k=1, \cdots, t \quad$ and $\quad g_{i j}(\mathbf{x}), i=1,2, \cdots, r, j=1,2, \cdots, s \quad$ are convex functions, $Q=\left(\bar{q}_{i j}\right), i=1, \cdots, r, j=1, \cdots, s$ is the matrix of constraint positive interval coefficients, $\overline{\mathbf{g}}=\left(\bar{\gamma}_{k}\right), k=1, \ldots, t, \overline{\mathbf{b}}=\left(\bar{b}_{i}\right), i=1, \ldots, m, \mathbf{x}=\left(x_{z}\right), z=1, \ldots, n$ are vectors of interval objective positive coefficients, interval right hand side and decision variables, respectively.

The aim of this paper is to extend the method of given in [8] and its modified form [7] for finding the upper bound and the lower bound of the range of the objective values in problem (1)-(2), by employing a two-level mathematical programming technique.

\section{The upper and lower bound problems}

First we consider some notations in presenting the interval of parameters and coefficients in problem (1)-(2). In general, each interval quantity $\bar{a}$ is denoted as $\left[a^{l}, a^{u}\right]$, where $a^{l}$ and $a^{u}$ are real numbers. Since the coefficients in objective function (1) are interval, so the objective value is interval. To find the bounds of interval objective value, suffices to find the lower bound and upper bound of the objective value of problem (1)-(2). Denote

$$
S=\left\{\left(\gamma_{k}, q_{i j}, b_{i}\right): \gamma_{k} \in \bar{\gamma}_{k}, q_{i j} \in \bar{q}_{i j}, b_{i} \in \bar{b}_{i}, k=1, \ldots, t, j=1, \ldots, s, i=1, \ldots, r\right\} .
$$

The values of $\gamma_{k}, q_{i j}, b_{i}$ that attain the smallest and largest objective value for $Z$ can be determined from mathematical programming problems

$$
\begin{gathered}
Z^{l}: \min _{\left(\gamma_{k}, q_{i j}, b_{i} \in S\right)} \min _{\mathbf{x}} Z=\sum_{k=1}^{t} \gamma_{k} f_{k}(\mathbf{x}) \\
\sum_{j=1}^{s} q_{i j} g_{i j}(\mathbf{x}) \leq b_{i}, i=1, \cdots, r, \\
\mathbf{x} \geq 0 .
\end{gathered}
$$

and

$$
Z^{u}: \max _{\left(\gamma_{k}, q_{i j}, b_{i} \in S\right)} \min _{\mathbf{x}} Z=\sum_{k=1}^{t} \gamma_{k} f_{k}(\mathbf{x})
$$




$$
\begin{gathered}
\sum_{j=1}^{s} q_{i j} g_{i j}(\mathbf{x}) \leq b_{i}, i=1, \cdots, r, \\
\mathbf{x} \geq 0 .
\end{gathered}
$$

The pair of mathematical programs (3)-(4) and (5)-(6) expresses the bound of the objective values. But these models are not solvable in the current form. In the next section we discuss how to transform these models to conventional programming.

\section{Finding upper and lower bound}

The interval of the objective values of problem (1)-(2) is obtained by giving its lower bound and upper bound. Now we discuss about obtaining the lower bound.

\section{Lower bound}

Clearly program (3)-(4) can be written as an equivalent problem below

$$
\begin{gathered}
Z^{l}: \min _{\mathbf{x}} Z=\sum_{k=1}^{t} \gamma_{k} f_{k}(\mathbf{x}) \\
\sum_{j=1}^{s} q_{i j} g_{i j}(\mathbf{x}) \leq b_{i}, i=1, \ldots, r, \\
\gamma_{k}^{l} \leq \gamma_{k} \leq \gamma_{k}^{u}, k=1, \ldots, t, \\
q_{i j}^{l} \leq q_{i j} \leq q_{i j}^{u}, i=1, \ldots, r, j=1, \ldots, s, \\
b_{i}^{l} \leq b_{i} \leq b_{i}^{u}, i=1, \ldots, r, \\
\mathbf{x} \geq 0 .
\end{gathered}
$$

In searching for the minimal value of the objective function, the parameter $\gamma_{k}, k=1, \ldots, t$ must reach its lower bound. Consequently we have

$$
\min _{\mathbf{x}} Z=\sum_{k=1}^{t} \gamma_{k}^{l} f_{k}(\mathbf{x})
$$

It is easy to see that the largest feasible region defined by inequality constraint $\sum_{j=1}^{s} q_{i j} g_{i j}(\mathbf{x}) \leq b_{i}$ in problem (7)-(8) appears when the interval parameter $b_{i}$ is equal to its upper bound and $q_{i j}$ is at its lower bound. Problem (7)-(8) then becomes

$$
\begin{gathered}
Z^{l}: \min _{\mathbf{x}} Z=\sum_{k=1}^{t} \gamma_{k}^{l} f_{k}(\mathbf{x}) \\
\sum_{j=1}^{s} q_{i j}^{l} g_{i j}(\mathbf{x}) \leq b_{i}^{u}, i=1, \ldots, r, \\
\mathbf{x} \geq 0,
\end{gathered}
$$

which is a conventional programming problem. The obtained objective value is a global optimum solution. Thus optimal solution $Z^{l}$ is the lower bound of the objective values of the interval convex programming. 


\section{Upper bound}

The upper bound of the objective value of the interval convex optimization problem (1)-(2) can be calculated from the two-level program of model (5)-(6). However, solving model (5)-(6) is not so straightforward because the outer program and inner program have different directions for optimization, i.e. one of them is maximization and another is minimization. So by writing the dual problem of inner program we transform minimization problem to maximization one. The Lagrangian dual problem of (5)-(6) is to maximize

$$
\beta(\mathbf{l}, \mathbf{d})=\inf \left\{\sum_{k=1}^{t} \bar{\gamma}_{k} f_{k}(\mathbf{x})+\sum_{i=1}^{r} \lambda_{i}\left(\sum_{j=1}^{s} \bar{q}_{i j} g_{i j}(\mathbf{x})-\bar{b}_{i}\right)-\sum_{z=1}^{n} \delta_{z} x_{z}\right\} .
$$

Since objective and constraint functions are convex and summation of convex functions is convex and so $\beta(\mathbf{l}, \mathbf{d})$ is convex. A necessary and sufficient condition for a minimum is that the gradient must vanish [2], that is:

$$
\sum_{k=1}^{t}-\gamma_{k} \frac{\partial f_{k}(\mathbf{x})}{\partial x_{z}}+\sum_{i=1}^{r} \lambda_{i}\left(\sum_{j=1}^{s}-\bar{q}_{i j} \frac{\partial g_{i j}(\mathbf{x})}{\partial x_{z}}\right)-\delta_{z}=0, z=1, \ldots, n .
$$

The dual form of the inner program in (5)-(6) can be written as follows:

$$
\begin{gathered}
\max _{\mathbf{x}, \mathrm{l}, \mathrm{d}} Z=\sum_{k=1}^{t} \bar{\gamma}_{k} f_{k}(\mathbf{x})+\sum_{i=1}^{r} \lambda_{i}\left(\sum_{j=1}^{s} \bar{q}_{i j} g_{i j}(\mathbf{x})-\bar{b}_{i}\right)-\sum_{z=1}^{n} \delta_{z} x_{z} \\
\sum_{k=1}^{t} \bar{\gamma}_{k} \frac{\partial f_{k}(\mathbf{x})}{\partial x_{z}}+\sum_{i=1}^{r} \lambda_{i}\left(\sum_{j=1}^{s} \bar{q}_{i j} \frac{\partial g_{i j}(\mathbf{x})}{\partial x_{z}}\right)-\delta_{z}=0, z=1, \ldots, n, \\
\lambda_{i}, \delta_{z} \geq 0, i=1, \ldots, r, z=1, \ldots, n .
\end{gathered}
$$

By Lagrangian duality if both problems are feasible then they both have optimal solutions and have the same objective value. In other words, models (5)-(6) can be reformulated as

$$
\begin{gathered}
Z^{u}=\max _{\left(\gamma_{k}, q_{i j}, b_{i}\right) \in S} \max _{\mathbf{x}, \mathbf{l}, \mathbf{d}} Z=\sum_{k=1}^{t} \bar{\gamma}_{k} f_{k}(\mathbf{x})+\sum_{i=1}^{r} \lambda_{i}\left(\sum_{j=1}^{s} \bar{q}_{i j} g_{i j}(\mathbf{x})-\bar{b}_{i}\right)-\sum_{z=1}^{n} \delta_{z} x_{z} \\
\sum_{k=1}^{t} \bar{\gamma}_{k} \frac{\partial f_{k}(\mathbf{x})}{\partial x_{z}}+\sum_{i=1}^{r} \lambda_{i}\left(\sum_{j=1}^{s} \bar{q}_{i j} \frac{\partial g_{i j}(\mathbf{x})}{\partial x_{z}}\right)-\delta_{z}=0, z=1, \ldots, n, \\
\lambda_{i}, \delta_{z} \geq 0, i=1, \ldots, r, z=1, \ldots, n .
\end{gathered}
$$

Now both the inner program and outer program have the same maximization operation, they can be merged in to a one-level program with constraints at the two -level considered at the same time :

$$
\begin{gathered}
Z^{u}=\operatorname{Max}_{\mathbf{x}, \mathbf{l}, \mathbf{d}} Z=\sum_{k=1}^{t} \gamma_{k} f_{k}(\mathbf{x})+\sum_{i=1}^{r} \lambda_{i}\left(\sum_{j=1}^{s} q_{i j} g_{i j}(\mathbf{x})-b_{i}\right)-\sum_{z=1}^{n} \delta_{z} x_{z}, \\
\sum_{k=1}^{t} \gamma_{k} \frac{\partial f_{k}(\mathbf{x})}{\partial x_{z}}+\sum_{i=1}^{r} \lambda_{i}\left(\sum_{j=1}^{s} q_{i j} \frac{\partial g_{i j}(\mathbf{x})}{\partial x_{z}}\right)-\delta_{z}=0, z=1, \ldots, n, \\
\gamma_{k}^{l} \leq \gamma_{k} \leq \gamma_{k}^{u}, k=1, \ldots, t, \\
q_{i j}^{l} \leq q_{i j} \leq q_{i j}^{u}, i=1, \ldots, r, j=1, \ldots, s, \\
b_{i}^{l} \leq b_{i} \leq b_{i}^{u}, i=1, \ldots, r, \\
\lambda_{i}, \delta_{z} \geq 0, i=1, \ldots, r, z=1, \ldots, n .
\end{gathered}
$$


To reach the maximal value of the objective function the interval parameters $\gamma_{k}, q_{i j}$ must reach their upper bound and $b_{i}$ must reach its lower bound. Thus we have

$$
\begin{gathered}
Z^{u}=\operatorname{Max}_{(\mathbf{x}, \mathbf{l}, \mathbf{d})} Z=\sum_{k=1}^{t} \gamma_{k}^{u} f_{k}(\mathbf{x})+\sum_{i=1}^{r} \lambda_{i}\left(\sum_{z=1}^{s} q_{i j}^{u} g_{i j}(\mathbf{x})-b_{i}^{l}\right)-\sum_{z=1}^{n} \delta_{z} x_{z}, \\
\sum_{k=1}^{t} \gamma_{k}^{u} \frac{\partial f_{k}(\mathbf{x})}{\partial x_{z}}+\sum_{i=1}^{r} \lambda_{i}\left(\sum_{j=1}^{s} q_{i j}^{u} \frac{\partial g_{i j}(\mathbf{x})}{\partial x_{z}}\right)-\delta_{z}=0, z=1, \ldots, n, \\
\lambda_{i}, \delta_{z} \geq 0, i=1, \ldots, m, z=1, \ldots, n .
\end{gathered}
$$

This model is conventional programming, and the obtained objective value is a global optimum solution. The optimal solution $Z^{u}$ is the upper bound of the objective values of the interval convex program. With $Z^{l}$ solved in (9)-(10), $\left[Z^{l}, Z^{u}\right]$ constitutes the interval that the objective values of the interval convex program lie.

\section{Numerical results} parameters:

Example 4.1. Consider the following convex programming with interval

$$
\begin{gathered}
Z=\min [1,2] x_{1}^{2} x_{2}^{2}+[3,4] x_{1}^{2}+[1,9] \\
\text { s.t. }[1,6] x_{1}^{4}+[0.5,1] x_{2}^{2} \leq[2,5], \\
{[2,3] x_{2}+[4,10] x_{1}^{2} x_{2}^{2} \leq[1,7],} \\
x_{1}, x_{2} \geq 0 .
\end{gathered}
$$

The upper bound of the objective value $Z^{u}$ according to model (19) -(20) can be formulated as:

$$
\begin{gathered}
Z^{u}=\max 2 x_{1}^{2} x_{2}^{2}+4 x_{1}^{2}+9+\lambda_{1}\left(6 x_{1}^{4}+x_{2}^{2}-2\right)+\lambda_{2}\left(3 x_{2}+10 x_{1}^{2} x_{2}^{2}-1\right)-\delta_{1} x_{1}-\delta_{2} x_{2} \\
\text { s.t. } 4 x_{1} x_{2}^{2}+8 x_{1}+24 x_{1}^{3} \lambda_{1}+20 x_{1} x_{2}^{2} \lambda_{2}-\delta_{1}=0, \\
4 x_{1}^{2} x_{2}+2 x_{2} \lambda_{1}+3 \lambda_{2}+20 x_{1}^{2} x_{2} \lambda_{2}-\delta_{2}=0, \\
\lambda_{1}, \lambda_{2}, \delta_{1}, \delta_{2} \geq 0 .
\end{gathered}
$$

By using LINGO, we obtain $x_{1}^{*}=0, x_{2}^{*}=0, Z^{u}=9$.

According to model (9) -(10), the lower bound of objective value $Z^{l}$ can be formulated as:

$$
\begin{gathered}
Z^{l}: \min x_{1}^{2} x_{2}^{2}+3 x_{1}^{2}+1 \\
\text { s.t. } x_{1}^{4}+0.5 x_{2}^{2} \leq 5 \\
2 x_{2}+4 x_{1}^{2} x_{2}^{2} \leq 7 \\
x_{1}, x_{2} \geq 0
\end{gathered}
$$


and $x_{1}^{*}=0, x_{2}^{*}=0, Z^{l}=1$. Combining these two results we conclude that the objective values of this interval convex programming lie in the range of $[1,9]$.

Example 4.2. Now consider the following problem

$$
\begin{gathered}
Z=\min [0,1] e^{x_{1}^{2}+x_{2}^{2}}+[1,2] x_{1}^{2}+[2,3] x_{2}^{4} \\
\text { s.t. }[1,2] x_{1}^{2}+[1,2] x_{2}^{2} \leq[1,4] \\
e^{x_{1}}+[1,3] x_{2} \leq[2,3] \\
x_{1}, x_{2} \geq 0 .
\end{gathered}
$$

The optimal solution of this problem is $x_{1}^{*}=0, x_{2}^{*}=0, Z=1$.

The upper bound of the objective value $Z^{u}$ according to model (19) -(20) can be formulated as:

$$
\begin{gathered}
Z^{u}: \max e^{x_{1}^{2}+x_{2}^{2}}+2 x_{1}^{2}+3 x_{2}^{4}+\lambda_{1}\left(2 x_{1}^{2}+2 x_{2}^{2}-1\right)+\lambda_{2}\left(e^{x_{1}}+3 x_{2}-2\right)-\delta_{1} x_{1}-\delta_{2} x_{2} \\
\text { st. } 2 x_{1} e^{x_{1}^{2}+x_{2}^{2}}+4 x_{1}+4 x_{1} \lambda_{1}+e_{1}^{x} \lambda_{2}-\delta_{1}=0, \\
2 x_{2} e^{x_{1}^{2}+x_{2}^{2}}+12 x_{2}^{3}+4 x_{2} \lambda_{1}+3 \lambda_{2}-\delta_{2}=0 . \\
\lambda_{1}, \lambda_{2}, \delta_{1}, \delta_{2} \geq 0 .
\end{gathered}
$$

By using MATLAB (fmincon), we obtain $x_{1}^{*}=0, x_{2}^{*}=0, Z^{u}=1$.

According to model (9)-(10), the lower bound of objective value $Z^{l}$ can be formulated as:

$$
\begin{gathered}
Z^{l}: \min x_{1}^{2}+2 x_{2}^{4} \\
\text { s.t. } x_{1}^{2}+x_{2}^{2} \leq 4, \\
e^{x_{1}}+x_{2} \leq 3, \\
x_{1}, x_{2} \geq 0 .
\end{gathered}
$$

and $x_{1}^{*}=0.9357, x_{2}^{*}=0.4511, Z^{l}=0.9584$. Combining these two results we conclude that the objective values of this interval convex programming lie in the range of $[0.9584,1]$.

\section{Conclusion}

In this paper we have generalized a numerical scheme to convex programming that objective function and constraint functions are convex.

Since the objective value is interval, we calculate the upper bound and lower bound of objective function where upper bound of programming is two-level problem. By using Lagrangian function we transformed a two-level problem to one-level. By numerical results, the proficiency of the given approach has been examined.

\section{References}

[1] S. A. Abass, An interval number programming approach for bilevel linear programming problem, International J. Management Science and Engineering Management, 
5(6) (2010) 461-464.

[2] M.S. Bazaraa, H.D. Sherali, C.M. Shetty, Nonlinear Programming: Theory and Algorithm, second ed., John Wiley and Sons, New York, 1993.

[3] M.F. Cao, G.H. Huang and L. He, An approach to interval programming problems with left-hand-side stochastic coefficients: An application to environmental decisions analysis, Expert Systems with Applications, 38(9) (2011) 11538-11546.

[4] H. Ishibuchi and H. Tanaka, Multiobjective programming in optimization of the interval objective function, European Journal of Operational Research, 48 (1990) 219-225.

[5] D. Kuchta, Ordering of Intervals and Optimization Problems with Interval Parameters, Central European J. Operation Research, 16 (2008) 307-316.

[6] K.K. Lai, S.Y. Wang, J.P. Xu, S.S. Zhu, Y. Fang, A class of linear interval programming problems and its application to portfolio selection, , IEEE Transactions on Fuzzy Systems, 10(6) (2002) 698 - 704.

[7] W. Li, X. Tian, Numerical solution method for general interval quadratic programming, Applied Mathematics and Computation, 202 (2008) 589-595.

[8] S.-T. Liu, R.-T. Wang, A numerical solution method to interval quadratic programming, Applied Mathematics and Computation, 189 (2007) 1274-1281.

[9] H.-C. Wu, The Karush-Kuhn-Tucker optimality conditions in an optimization problem with interval-valued objective function, European Journal of Operational Research, 176(1) (2007) 46-59.

[10] H. -C. Wu, On interval-valued nonlinear programming problems, J. Math. Anal. Appl. 338 (2008) 299ï3161\%? 